\title{
Pulmonary radioaerosol mucociliary clearance in primary ciliary dyskinesia
}

\begin{abstract}
To the Editor:
Primary ciliary dyskinesia (PCD) is a genetic condition affecting one in 10000-40000 people from birth [1]; cilia fail to beat, and the airway clearance of mucus and debris is severely impaired. If untreated, this results in progressive lung infection leading to bronchiectasis and ultimately respiratory failure. Additionally, delayed diagnosis has implications for genetic counselling, appropriate management of glue ear and fertility advice. Early diagnosis and appropriate treatment are believed to improve outcome. The diagnosis of PCD is highly specialised and results can remain inconclusive, despite state of the art equipment and diagnostic techniques. A European consensus statement $[2,3]$ highlighted that there is no "gold-standard" diagnostic test; diagnosis requires expert review of clinical history and screening tests (nasal nitric oxide measurement) alongside analysis of ciliary function and ultrastructure [2]. It is recommended that ciliary activity of respiratory epithelial cells obtained by nasal or bronchial brushing is recorded using a high-speed video camera mounted on a microscope. The images are played back in slow motion to analyse ciliary beat pattern (CBP) and frequency (CBF). Transmission electron microscopy (TEM) is used to assess ciliary ultrastructure [4]. Diagnostic uncertainty can be caused by secondary damage of the epithelium during sampling or due to infection or inflammation of epithelia; this damage can lead to abnormalities of ultrastructure, CBF and CBP. Furthermore, diagnosis is hindered by normal ciliary ultrastructure in $3-30 \%$ of cases of $\operatorname{PCD}[5,6]$. To improve diagnostic certainty, a variety of further investigations can be employed [2], including reanalysis of CBF, CBP and TEM following culture of the cells at an air-liquid interface [7, 8], or using immunofluorescence microscopy [9] to identify ciliary proteins. A single-centre study has previously reported the use of pulmonary radioaerosol mucociliary clearance (MCC) in the diagnosis of PCD [10]. The method is based on clearance patterns after the inhalation of a radioaerosol tracer. It provides a whole-lung functional test for pulmonary radioaerosol MCC. The investigation is noninvasive and has been used in thousands of patients with other lung diseases, as young as $\sim 5$ years. The authors reported that MCC was an effective noninvasive functional test for PCD [10] but the study was preliminary, and the feasibility of this complex technique and interpretation of data have not been assessed in other centres. We therefore conducted a study to replicate the results using a standardised protocol. This study was approved by the National Research Ethics Service (South Central committee 11/ SC/0192) and all subjects gave written informed consent.
\end{abstract}

We assessed pulmonary radioaerosol MCC in six adult patients previously diagnosed with "classical" PCD (table 1) according to European Respiratory Society consensus guidelines [2] compared with four healthy controls. PCD and control groups were similar in age and sex distribution but PCD patients had impaired lung function (mean FEV1 74\% (range 36-105\%) predicted in PCD patients and 97\% (range 93-100\%) predicted in controls) (table 1). Participants inhaled nebulised technetium-99m labelled nanocoll (GE Healthcare, Little Chalfont, UK) via a DeVilbiss 646 nebuliser (DeVilbiss Healthcare, Somerset, PA, USA) while breathing to ensure the best deposition in the central airways, as described previously [11]. Planar

TABLE 1 Primary ciliary dyskinesia subject characteristics

\begin{tabular}{|c|c|c|c|c|c|c|}
\hline Subject & Age years & HSV findings & TEM findings & nNO ppb & FEV1 L & FEV $1 \%$ predicted \\
\hline 1 & 19.0 & Static and twitching & Normal & 100 & 3.74 & 84 \\
\hline 2 & 16.8 & Static & ODA defect & 86 & 2.95 & 67 \\
\hline 4 & 61.1 & Static and twitching & ODA and IDA defect & 57 & 0.6 & 36 \\
\hline 5 & 43.4 & Static & ODA and IDA defect & 30 & 1.82 & 71 \\
\hline 6 & 18.9 & $\begin{array}{l}\text { Dyskinetic and } \\
\text { uncoordinated }\end{array}$ & $\begin{array}{c}\text { Axonemal disorganisation } \\
\text { and absent IDAs }\end{array}$ & 40 & 3.12 & 80 \\
\hline
\end{tabular}

HSV: high-speed video microscopy; TEM: transmission electron microscopy; nNO: nasal nitric oxide; FEV1: forced expiratory volume in 1 s; ODA: outer dynein arm; IDA: inner dynein arm. 


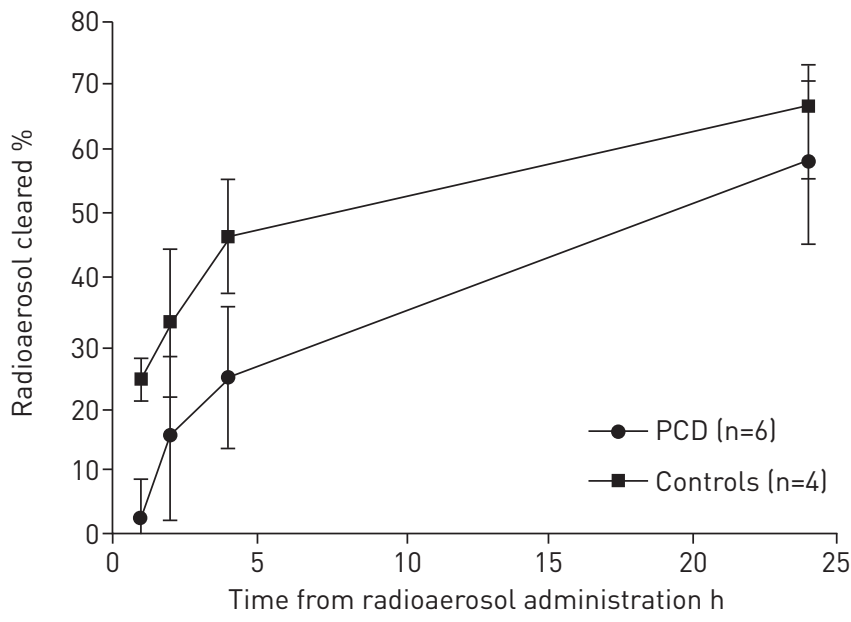

FIGURE 1 Mean percentage of radioaerosol cleared at $1,2,4$ and $24 \mathrm{~h}$ in the primary ciliary dyskinesia (PCD) and control groups. Error bars represent $95 \%$ confidence intervals.

gamma camera images were taken at time $0,1,2,4$ and $24 \mathrm{~h}$ on a dual-head GE Infinia gamma camera (GE Systems, Milwaukee, WI, USA). A single-photon emission computed tomography/computed tomography scan and krypton-81 ventilation images were also obtained to characterise the initial distribution, allow attenuation correction and define the lung outline to assist interpretation of clearance data [12]. Geometric mean image data were interpreted after applying attenuation correction. Image analysis was carried out blind to patient group.

The mean percentage radioaerosol cleared was significantly greater in the control than in the PCD group at both $1 \mathrm{~h}$ (mean $24.0 \%$ (95\% CI 3.4\%) versus $2.3 \%$ (95\% CI 6.1\%)) and $4 \mathrm{~h}(46.3 \%(95 \%$ CI $8.7 \%)$ versus $24.2 \%$ (95\% CI 11.1\%)) (fig. 1). By 24 h, clearance was similar in the two groups $(66.2 \%$ (95\% CI $7.1 \%$ ) versus $57.9 \%$ (95\% CI $12.8 \%)$ ). The technique was well tolerated by the subjects and there were no adverse events. Cooperation is needed from participants and it is unlikely that the test would be possible in preschool children.

Our study adds to the limited data that pulmonary radioaerosol MCC might be a useful diagnostic adjunct for diagnosing PCD. The expertise required to conduct the studies and interpret the data are likely to limit it to specialist nuclear medicine centres. The normal clearance of the aerosol by $24 \mathrm{~h}$ demonstrates alternative methods of airway clearance in PCD than MCC, including chest clearance physiotherapy (the subjects were advised to undertake their normal physiotherapy regimen between the 4- and 24-h images), cough and macrophage activity. It provides reassurance of the safety of this radioaerosol technique. Further data are required to confirm whether the technique will be useful for differentiating patients with considerable secondary ciliary damage or for diagnosing patients with "atypical PCD" (e.g. normal ciliary ultrastructure).

0 @ERSpublications

Pulmonary radioaerosol mucociliary clearance is reduced in PCD patients and might prove useful in diagnosis http://ow.ly/uP5Vg

Woolf T. Walker ${ }^{1,2}$, Aneurin Young ${ }^{1,3}$, Michael Bennett ${ }^{1,3}$, Matthew Guy ${ }^{1,4}$, Mary Carroll ${ }^{1,2}$, John Fleming ${ }^{1,4}$, Joy Conway ${ }^{1,3,5}$ and Jane S. Lucas ${ }^{1,2}$

${ }^{1}$ NIHR Southampton Respiratory Biomedical Research Unit, University of Southampton and University Hospital Southampton NHS Foundation Trust, Southampton, UK. ${ }^{2}$ Primary Ciliary Dyskinesia Centre, University Hospital Southampton NHS Foundation Trust, Southampton, UK. ${ }^{3}$ Academic Unit of Clinical and Experimental Sciences, Faculty of Medicine, University of Southampton Faculty of Medicine, Southampton, UK. ${ }^{4}$ Dept of Medical Physics and Bioengineering, University Hospital Southampton NHS Foundation Trust, Southampton, UK. ${ }^{5}$ Faculty of Health Sciences, University of Southampton, Southampton, UK.

Correspondence: Jane S. Lucas, Academic Unit of Clinical and Experimental Sciences (Mail Point 803), University of Southampton Faculty of Medicine, University Hospital Southampton NHS Foundation Trust, Tremona Road, Southampton, SO16 6YD, UK. E-mail: jlucas1@soton.ac.uk

Received: Jan 152014 | Accepted after revision: March 182014 | First published online: May 22014

Conflict of interest: Disclosures can be found alongside the online version of this article at erj.ersjournals.com

\section{References}

1 Kuehni CE, Frischer T, Strippoli MP, et al. Factors influencing age at diagnosis of primary ciliary dyskinesia in European children. Eur Respir J 2010; 36: 1248-1258. 
2 Barbato A, Frischer T, Kuehni CE, et al. Primary ciliary dyskinesia: a consensus statement on diagnostic and treatment approaches in children. Eur Respir J 2009; 34: 1264-1276.

3 Bush A, Chodhari R, Collins N, et al. Primary ciliary dyskinesia: current state of the art. Arch Dis Child 2007; 92: 1136-1140.

4 Lucas JS, Walker WT, Kuehni CE, et al. Primary ciliary dyskinesia. In: Cordier J-F, ed. Orphan Lung Diseases. Eur Respir Monogr 2011; 54: 201-217.

5 Jorissen M, Willems T, Van der Schueren B, et al. Ultrastructural expression of primary ciliary dyskinesia after ciliogenesis in culture. Acta Otorhinolaryngol Belg 2000; 54: 343-356.

6 Shoemark A, Dixon M, Corrin B, et al. Twenty-year review of quantitative transmission electron microscopy for the diagnosis of primary ciliary dyskinesia. J Clin Pathol 2012; 65: 267-271.

7 Hirst RA, Rutman A, Williams G, et al. Ciliated air-liquid cultures as an aid to diagnostic testing of primary ciliary dyskinesia. Chest 2010; 138: 1441-1447.

8 Jorissen M, Willems T, Van der Schueren B. Ciliary function analysis for the diagnosis of primary ciliary dyskinesia: advantages of ciliogenesis in culture. Acta Otolaryngol 2000; 120: 291-295.

9 Omran H, Loges NT. Immunofluorescence staining of ciliated respiratory epithelial cells. Methods Cell Biol 2009; 91: 123-133.

10 Marthin JK, Mortensen J, Pressler T, et al. Pulmonary radioaerosol mucociliary clearance in diagnosis of primary ciliary dyskinesia. Chest 2007; 132: 966-976.

11 Bennett WD, Laube BL, Corcoran T, et al. Multisite comparison of mucociliary and cough clearance measures using standardized methods. J Aerosol Med Pulm Drug Deliv 2013; 26: 157-164.

12 Fleming JS, Conway JH, Majoral C, et al. The use of combined single photon emission computed tomography and X-ray computed tomography to assess the fate of inhaled aerosol. J Aerosol Med Pulm Drug Del 2011; 24: 49-60.

\title{
Severe influenza A H7N9 pneumonia with rapid virological response to intravenous zanamivir
}

\author{
To the Editor:
}

In March 2013, a novel influenza A H7N9 virus of avian origin was reported to cause severe pneumonia in mainland China [1-3]. While the great majority of patients were treated with oral oseltamivir, impaired gastrointestinal absorption in critically ill patients, the lack of virological response among patients requiring extracorporeal membrane oxygenation (ECMO) and detection of an amino acid change (arginine to lysine at residue 292 in N2) in the viral neuraminidase associated with drug resistance in two H7N9 strains are major concerns $[1,4]$. Here, we report a case of severe pneumonia due to avian influenza A H7N9 requiring ECMO support; there was rapid clinical and virological response following intravenous zanamivir therapy.

On November 21, 2013, in Hong Kong, China, a 36-year-old Indonesian female began to have fever, malaise and cough. She had been previously healthy except for a history of vitiligo. She sought medical advice from two clinics and was given symptomatic treatment. She attended the accident and emergency department of a regional hospital in Hong Kong on November 27 when the symptoms deteriorated. Her temperature was $40.0^{\circ} \mathrm{C}$, blood pressure was $120 / 63 \mathrm{mmHg}$, pulse was 116 beats $\cdot \mathrm{min}^{-1}$, respiratory rate was 18 breaths $\cdot \min ^{-1}$ and oxygenation was $97 \%$ while breathing ambient air. A chest radiograph showed right lower zone consolidation.

After admission, there was persistent fever, rapid progression of the radiological infiltrates to both lungs and development of parapneumonic effusion despite treatment with intravenous amoxicillin-clavulanate, ceftriaxone, azithromycin and oral oseltamivir (75 mg twice daily, total of two doses given). Endotracheal intubation and mechanical ventilation were required on November 29. Desaturation worsened and she was put on ECMO on November 30. Initial microbiological workup including sputum culture, blood culture, urine for Legionella and pneumococcal antigens, two nasopharyngeal swabs for influenza A and B antigens, and tracheal aspirate for human metapneumovirus, Mycoplasma pneumoniae, Chlamydophila pneumoniae and Mycobacterium tuberculosis by PCR were negative.

In view of the rapid disease progression, the respiratory specimens were subjected to testing for avian influenza viruses [5]. PCR identified presence of H7N9 in all the respiratory specimens (throat swab, nasopharyngeal swabs and aspirates, tracheal aspirate, and pleural fluid) collected since hospitalisation. No H7N9 was detected in urine or rectal swabs. PCR for influenza A subtypes H1, H3 and H5 of the nasopharyngeal swabs and tracheal aspirates was negative. Therapy with intravenous zanamivir $600 \mathrm{mg}$ 